thiamazole $30 \mathrm{mg}$ daily improved all the symptoms, including meralgia paraesthetica.

A year later, the left lateral cutaneous nerve of the thigh sensory conduction velocity had improved to $58.8 \mathrm{~m} / \mathrm{s}$ with normal amplitude $(3 \cdot 6 \mu \mathrm{V})$.

To discover the frequency of localised sensory disturbances in hyperthyroidism, we studied clinically 20 patients with Graves' disease, including these two cases (10 hyperthyroid patients, 10 euthyroid, after treatment). Symptoms consistent with mononeuropathy such as localised sensory disturbance or muscle weakness, were present in nine $(45 \%)$. These symptoms were dysaesthesia, paraesthesia or hypoaesthesia on fingers in five patients, hypoaesthesia or dysaesthesia on the lateral aspects of the thigh in seven patients, and bilateral foot drop in one patient (case 1). Tinel's sign of median nerve was present in seven of 10 hyperthyroid patients and five of 10 euthyroid patients. A positive Tinel's sign was found in $60 \%$ of thyrotoxic patients but also in $14.5 \%$ of 282 normal controls (Chi-square $=23.6, \mathrm{p}<0.00001$ ).

Our two cases demonstrated a combination of mononeuropathy and thyrotoxicosis. These mononeuropathies were confirmed by nerve conduction studies and improved following treatment for thyrotoxicosis. In addition, the denervation findings and low amplitude evoked reponses without conduction block of the peroneal nerve in case 1 suggest mono-axonopathy associated with thyrotoxicosis. The dissociation between complete foot drop and no conduction block of the peroneal nerve supports this possibility.

In euthyroid patients, the frequency of both the symptoms and Tinel's sign diminished with the duration of treatment.

In a detailed electrophysiological study ${ }^{3}$ of patients with thyrotoxicosis, loss of functioning motor units with normal conduction velocities demonstrated motor neuron dysfunction and the remarkable capacity of motor neurons to resume normal function. Individual nerves are more sensitive to mechanical damage if a generalised peripheral neuropathy is present. ${ }^{4}$ It seems likely to us that the fragility of nerve axons associated with hyperthyroidism predisposes to mononeuropathies.

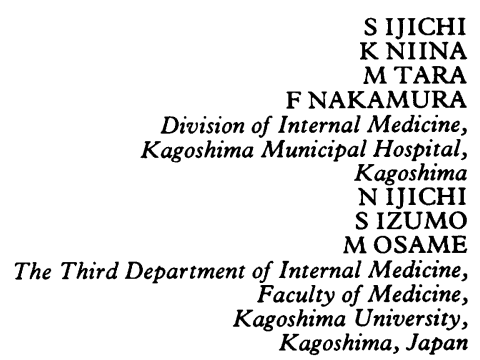

Correspondence to: The Third Department of Internal Medicine, Faculty of Medicine, KagoInternal Medicine, Faculty of Medicine, Kago890, Japan

1 Feibel JH, Campa JF. Thyrotoxic neuropathy Basedow's paraplegia). J Neurol Neurosurg sychiatry 1976;39:491-7.

2 Florin T, Walls RS. Neurological complications of thyrotoxicosis in the elderly. Ann Neurol 1984;15:608.

3 McComas AJ, Sica REP, McNabb AR, Goldberg WM, Upton ARM. Evidence for reversible motoneurone dysfunction in thyrotoxicosis. J Neurol Neurosurg Psychiatry 1974;37:548-58.

4 Stewart JD, Aguayo AJ. Compression and entrapment neuropathies. In: Dyck PJ, Thomas PK, Lambert EH, Bunge R, eds. Peripheral Neuropathy, ed 2. Philadelphia: WB Saunders, 1975;1435-57.
Correction:

This letter was printed in the August issue with only one MRI image.

\section{MRI of thoracic cord in tropical spastic paraparesis}

Tropical spastic paraparesis (TSP) is a disease occurring in Afro-Caribbeans following HTLV-1 retro-virus infection. There is some evidence that the geographical and ethnic distribution of HTLV-1 illness is even wider and HTLV-1 associated myelopathy (HAM) in Japan is probably the same disorder. Abnormalities are found on MRI of the brain in both $\mathrm{TSP}^{23}$ and HAM. ${ }^{4}$ High signal areas are found in the brain similar to those in multiple sclerosis (MS), though they tend to be less extensive. The thoracic cord (on which the brunt of the pathological process falls) has been examined in only three patients, one of whom had atrophy. ${ }^{2}$ Since the clinical picture of TSP may resemble that of progressive MS we have made a systematic comparison of the MRI characteristics of the thoracic cord in the two conditions.

Nine patients with TSP who were born in the Caribbean were compared with an age and sex matched group of European white patients with clinically definite $\mathrm{MS},{ }^{5}$ all of whom had a progressive spastic paraparesis Disability was scored using the Kurtzke Disability Status Scale. ${ }^{6}$ The patients with TSP were anti-HTLV1 positive and had HTLVgenome integrated into leucocyte DNA Eight were female. The mean age was 53 years (range 43-65 years), the mean symptom duration was 12 years (range $1 \cdot 5-23$ years), and the mean Kurtzke disability score was seven (range five to eight). The mean age of the MS patients was 42 years (range 35 to 53 years), the mean symptom duration was 11 years (range seven to 17 years), and the mean Kurtzke disability score was five (range 4 to

6). The spine was imaged by a Picker $0.5 \mathrm{~T}$ superconducting machine with $\mathrm{T} 1$ weighted $\left(\mathrm{SE}_{500} 40\right) 5 \mathrm{~mm}$ contiguous parasagittal slices using a surface coil. All MS patients and five TSP patients had additional T2-weighted sequences $\left(\mathrm{SE}_{150080}, 5 \mathrm{~mm}\right.$ contiguous parasagittal slices) to detect abnormal signal. Images were reported without knowledge of the individual diagnosis by one of the authors (EPGH du B)

Atrophy of the thoracic cord was seen in six of nine patients with TSP and five of nine patients with MS. Three of five patients with TSP who had T2-weighted images of thoracic cord had diffuse high signal and all three had atrophy (fig). Five of nine with MS had high signal return on T2 weighted images, one of whom did not have atrophy. The pattern of high signal was diffuse in two and focal or patchy in three (fig).

These results confirm the previous MRI finding of atrophy in the thoracic cord in a proportion of patients with TSP. However, a similar degree of atrophy is seen as frequently in patients with MS who had a progressive spastic paraparesis, a finding compatible with pathological studies where cord atrophy is present in $72 \%$ of patients with MS at necropsy. ${ }^{7}$ There was some difference in the pattern of high signal seen in the two groups, with more diffuse and uniform high signal in TSP and focal or patchy high signal in MS However, these differences in the MRI findings are slight and a reliable distinction between the two conditions cannot be made on these grounds.
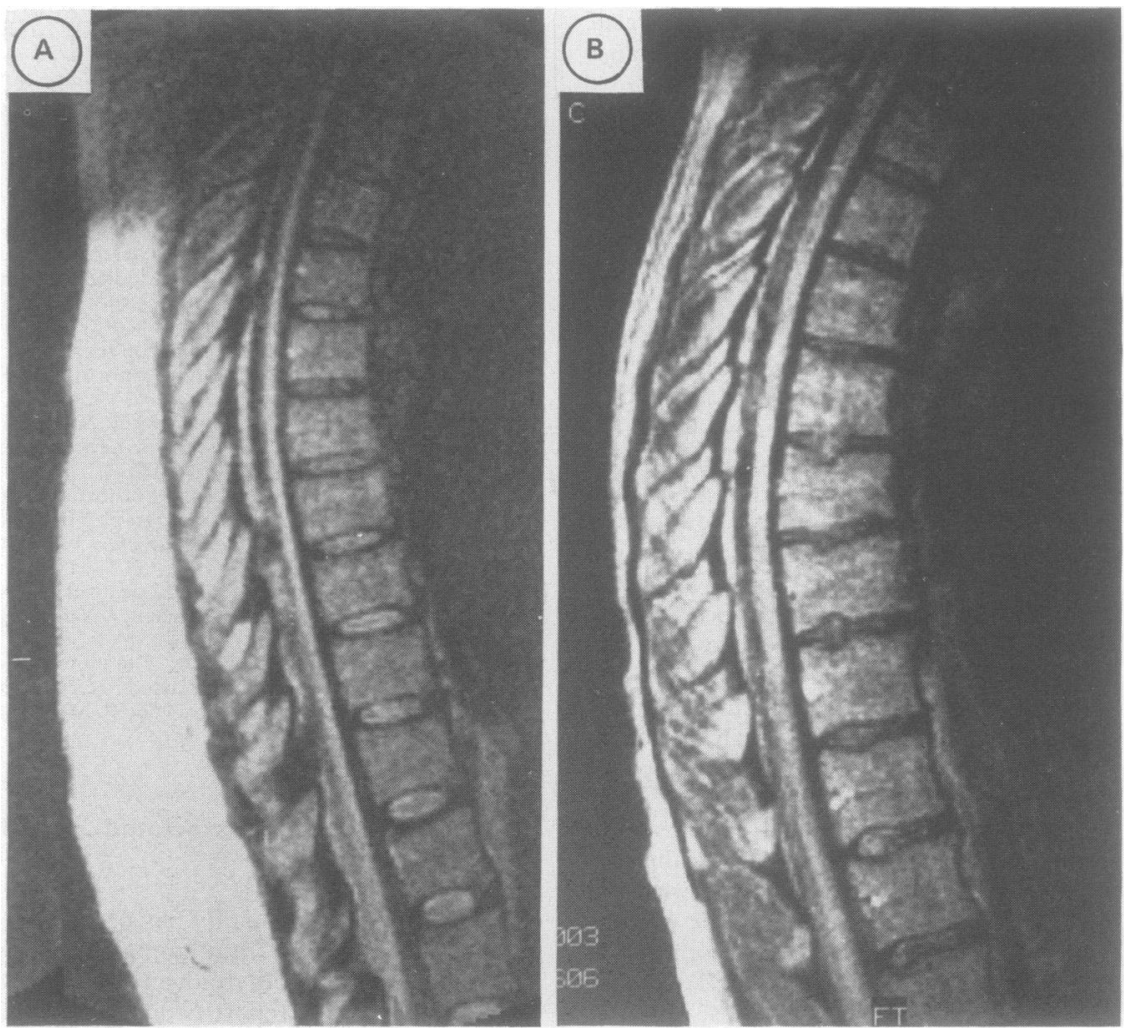

Figure The MRI on the left shows diffuse high signal with atrophy of the thoracic cord in TSP. The right shows the patchy high signal typically seen in $M S$. ( $S E_{t s 008}, 5 \mathrm{~mm}$ sagittal slices). 
Correspondence to: Dr Allan G Kermode, NMR Research Group, Insitute of Neurology, Queen Square, London WC1N 3BG, United Kingdom

The Multiple Sclerosis Work Group is supported by the Multiple Sclerosis Society of Great Britain and Northern Ireland, and by the Medical Research Council.

1 Cartier-Rovirosa L, Mora C, Araya F, et al. HTLV1 positive spastic paraparesis in a tem HTLV1 positive spastic paraparesis

2 Cruickshank JK, Rudge P, Dagleish AG, et al. Tropical spastic paraparesis and Human $\mathrm{T}$ Tropical spastic paraparesis and Human T-
cell Lymphotropic Virus type 1 in the United Kingdom. Brain 1989;112:1057-90.

3 Newton M, Cruickshank JK, Miller D, et al. Antibodies to HTLV-1 in West Indian born UK resident patients with spastic paraparesis. Lancet 1987; i:415-6.

4 Kira J, Minato S, Itoyama Y, Goto I, Kato M, Hasuo K. Leukoencephalopathy in HTLV-1associated myelopathy: MRI and EEG data. Neurol Sci 1988;87:221-32.

5 Poser CM, Paty DW, Scheinberg L, et al. New diagnostic criteria for multiple sclerosis: guidelines for research protocols. Ann Neurol 1983;13:227-31.

6 Kurtzke JF. Further notes on disability evalua tion in multiple sclerosis with scale modification in multiple sclerosis with scale modif

7 Ikuta F, Zimmerman HM. Distribution of kuta F, Zimmerman HM. Distribution of
plaques in seventy autopsy cases of multiple sclerosis in the United States. Neurology $1976 ; 26$ (6, pt 2):26-8.

\section{(1) \\ MATTERS ARISING}

Non-vascular aetiology of lacunar syndromes

We have read with interest the paper by Anzalone and Landi ${ }^{1}$ on non-ischaemic causes of lacunar syndromes. From 31 August 1985 to 31 October 1989, we studied prospectively our patients from the stroke clinic that included several cases of lacunar infarctions and lacunar syndromes. We have found several cases of lacunar syndromes due to non-vascular aetiology. The main cause of lacunar syndrome in our patients was neurocysticercosis.

Some of these cases have been reported elsewhere. ${ }^{2-5}$ Our series include 12 patients aged 18 to 57 years, mean 34.5 years. During the last four years 733 patients attended our stroke clinic and from these we found 114 cases with lacunar syndrome. The twelve cases due to neurocysticercosis are $8.3 \%$ of lacunar syndromes.

In two patients the lacunar syndrome (ataxic hemiparesis in both) were produced by parenchymal brain cysticercosis, ${ }^{2}$ in the remaining patients the lacunar syndrome was produced by cerebral infarction (lacunar infarction). In most cases it was located in the capsular area and in only two patients was the lesion located in the subcortical area. These infarctions were associated with subarachnoid cysticerci. It has been well recognised that subarachnoid cysticerci usually induced subacute, chronic or recurrent meningitis with abnormal thickening of leptomeninges at the base of the skull and inflammatory entrapment of blood vessels around the circle of Willis. The histopathological findings in these vessels usually include fibrosis of the media and endothelial hyperplasia and occasionally complete occlusion.

As in the series of Anzalone and Landi ${ }^{1}$ a past history of arterial hypertension was uncommon in our series.

The lacunar syndromes in the present series included sensorimotor syndrome in five patients, pure motor hemiparesis in four and in three patients ataxic-hemiparesis. Two cases had cysticerci in brain parenchyma, suprasellar in five, one interpeduncular, one insular, one meningeal and diffuse arachnoiditis in two cases.

Cerebral angiography was performed in five patients, and evidence of vasculitis was found in three patients.

Cysticercosis is not usually considered in the differential diagnosis of lacunar syndromes, this becomes important in areas of the world where cysticercosis is endemic.

We agree with Anzalone and Landi ${ }^{1}$ with the concept that early CT scanning must be performed in patients with lacunar syndrome, particularly if the patient is young, normotensive or resident in countries where neurocysticercosis is a frequent health problem. In the latter we would strongly recommend the routine examination of cerebrospinal fluid including immune reactions for cysticercosis.

FERNANDO BARINAGARREMENTERIA Stroke Clinic,

Instituto Nacional de Neurologia Y Neurocirugia, Mexico 22

1 Anzalone N, Landi G. Non-ischaemic causes of lacunar syndromes: prevalence and clinical findings. $J$ Neurol Neurosurg Psychiatry 1989;52:1188-90.

2 Barinagarrementeria F, Del Brutto $\mathrm{OH}$, Otero E. Ataxic hemiparesis from cysticercosis. Arch Neurol 1988;45:246.

3 Barinagarrementeria F, Del Brutto OH. Ataxic hemiparesis from parenchymal brain cysticercosis. J Neurol 1988;235:325.

4 Barinagarrementeria F, Del Brutto $\mathrm{OH}$ Neurocysticercosis and pure moto hemiparesis. Stroke 1988;19:1156-8.

5 Barinagarrementeria F, Del Brutto $\mathrm{OH}$ Lacunar Syndrome due to neurocysticercosis. Arch Neurol 1989;46:415-7.

\section{Crying and laughing after brain damage}

We doubt that Allman's suggestion' will find much support, yet the problem he considers is a real one. It is not only a question of facilitating communication. An important aspect is the need for a term that, like most other medical terms, can be used in various parts of speech without recourse to cumbersome circumlocution. The indispensable need, however, is for a term that recognises the essential feature of the phenomenon, namely that it is a disorder of the mechanisms of expression of emotion, and not of emotion or affect itself. This is what is wrong with the suggestion "emotionalism".

Perhaps the solution for this problem of nomenclature demands a lateral flight of fancy. The vocabulary of medicine is predominantly Greek. On the whole, this poses no problems, indeed it helps to avoid the contamination of meaning that often attends the use of English expressions through their vernacular connotations. The traditional symbol of the expression of emotion is the ancient Greek diptych of the theatrical laughing and crying masks, known as prosopeion. This suggests dysprosopeia, allowing the equally simple adjective dysprosopeic. Where Sigmund Freud has trod, we surely dare to go.

PETER EAMES DEMETRIOS PAPAKOSTOPOULOS Burden Neurological Institute Bristol BS16 1QT

1 Allman P. Crying and laughing after brain damage: a confused nomenclature: $J$ Neurol Neurosurg Psychiatry 1989;52:1439-40.

\section{BOOK REVIEWS}

Therapy of Parkinson's Disease. Neurologic Disease and Therapy Series 5). Edited by WC KOLLER AND G PAULSON (Pp 583 Illustrated; Price $\$ 125.00$ (US and Canada), $\$ 150.00$ (All other countries.) New York: Marcel Dekker Inc. 1990. ISBN 0-82478219-4

Having enjoyed reading and commending Koller's Handbook of Parkinson's Disease last year, I looked forward to learning more about therapy from this new book edited jointly with Paulson. Their preface reminds us, perhaps a little unkindly, that few of the contributing authors are old enough to remember patients with end-stage Parkinson's disease "who lay in sawdust" ... or those given levodopa in whom we "witnessed dramatic changes as patients in Stages III or IV became completely functional". Very true, but I am not sure we would all agree with Koller's introductory remark that "the types of therapy have dramatically increased pari passu ... due in large part to the discovery of MPTP model of parkinsonism.' The contents show 69 contributors in serried ranks, amongst whom are included almost all of the USA doyens as well as a selection of Europeans.

The five main sections cover Assessment and measurement of symptoms and signs, Pharmacologic agents, Surgical approaches, Diet in therapy, and Other therapeutic approaches.

The result is a very useful and comprehensive survey of treatment which is up-to-date, well referenced and authoritative. Modern techniques range from apomorphine, a rebirth of stereotaxic thalamotomies, appraisal of neurotransplantation, levodopa infusions and, to be right up to the minute the DATATOP trial of selegiline and tocopherol; all are reviewed in detail.

There are however faults. In a fact laden text, good writing and thereby easy reading are just as important as in a more discursive series of essays. And here the book disappoints, despite the publisher's attractive printing format illuminated by clear illustrations and diagrams. Many contributors have careened the edifice of rational, reflective prose in favour of an almost obsessive devo- 